\title{
Balanceo de un plan de estudios mediante la aplicación del algoritmo COMSOAL
}

\section{Rolling a Plan of studies through the application of the COMSOAL algorithm}

\author{
Heidy Mejía Ávila \\ Ms. C en Ingeniería Industrial de la Universidad de Puerto Rico, Recinto de Mayagüez. \\ Profesor Titular del Programa de Ingeniería Industrial de la Universidad Autónoma del Caribe. hmejia@uac.edu.co. \\ Marjorie Galofre Vásquez \\ Candidato a Ms. C. en Ingeniería Industrial de la Universidad del Norte \\ Profesor Asistente del Programa de Ingeniería Industrial de la Universidad Autónoma del Caribe. mgalofre@uac.edu.co. \\ Norberto Acuña Molina \\ Magister en Ingeniería Ambiental de la Universidad Nacional de Colombia. Profesor Asistente de la Universidad Autónoma \\ del Caribe del Programa de Ingeniería Industrial. nacuna@uac.edu.co \\ Recibido: oct 6 de 2012 \\ Aceptado: oct. .26 de 2012
}

\begin{abstract}
RESUMEN
Este artículo, presenta un estudio investigativo, que tuvo como población un programa de Ingeniería Industrial de la costa Atlántica, que requería seguir los lineamientos exigidos para su renovación curricular, donde debía desarrollar un plan de estudios acorde a las necesidades del medio y a la demanda del sector productivo, ya que se había detectado sobrecarga académica que problematizaban la dinámica de la renovación curricular. Para evitar el inconformismo generado por la sobrecarga académica se planteó evaluar la distribución de las asignaturas en los semestres y se pudo trabajar en equipo de tres personas, para establecer una analogía con el Problema de Balanceo de líneas de ensamble, el cual fue resuelto mediante el algoritmo COMSOAL. Con esta herramienta se generó, por parte de la investigación, una alternativa de rediseño, y se compararon sus eficiencias.
\end{abstract}

Se presenta la alternativa de esta herramienta como medio para la optimización de los programas de estudio mediante la detección temprana de debilidades o falencias, así como el análisis de la pertinencia de los prerrequisitos en el proceso de diseño.

Palabras Clave: Plan de estudios, balanceo de líneas de ensamble, COMSOAL, eficiencia.

\begin{abstract}
The Industrial Engineering program following the guidelines required for curricular renewal developed a curriculum tailored to the needs of the environment and the productive sector demand. To avoid the inconsistency generated by the academic overload it was planed to evaluate the distribution of subjects in the semester, it was stablish an analogy with the problem of balancing assembly lines, which was resolved using COMSOAL algorithm generating an alternative of redesign, and comparing their efficiencies.
\end{abstract}

It's presented as the alternative to this tool as a mean of optimizing programs of study through the early detection of weaknesses and shortcomings, and the analysis of the relevance of the prerequisites in the design process.

Key words: Academic curriculum, Assembly line balancing, COMSOAL, efficiency. 


\section{Introducción}

El programa de Ingeniería Industrial de la Universidad Autónoma del Caribe, como todo programa de educación superior en el país; está regido por el Ministerio de Educación Nacional, su objetivo es formar las competencias al futuro profesional que le permita desempeñarse en su ámbito laboral, acorde a los requerimientos y la dinámica del contexto socio-económico de la región y el país. Es "per se" responsabilidad de la Institución la renovación permanente de estos programas, en la búsqueda de ofrecer Profesionales altamente competitivos.

El plan de estudios ofertado, debe desarrollar las competencias y saberes de los estudiantes y transformarlos en profesionales capacitados para atender la problemática del entorno, para el cumplimiento de esta formación se agrupan los cursos en cinco áreas del saber: básica, básica de ingeniería, profesional, sociohumanística y electivas; el estudiante es promocionado mediante el alcance de las competencias de cada curso en una cadena de prerrequisitos.

Al estructurar un plan de estudios, sea este el caso del programa de Ingeniería Industrial de la Universidad Autónoma del Caribe, en un proceso de rediseño curricular es importante tener en cuenta el balanceo de la carga académica en cada uno de los períodos o niveles, dada su directa relación con el proceso enseñanzaaprendizaje. La coordinación de Bienestar Universitario, detectó el aumento de la repitencia en los semestres de mayor carga y por ende el descontento de los estudiantes al llegar a cursar estos semestres.

Haciendo un símil con el balanceo de líneas de ensamble se busca la ubicación de los cursos de manera tal que en cada uno de los períodos académicos se mantenga una carga horaria promedio, garantizando el menor desgaste y el cumplimiento de los objetivos de los programas; para esto se seleccionó la aplicación de la técnica heurística de optimización COMSOAL (Arcus 1996).

\section{Referentes Teóricos}

La línea de ensamble, tal como lo enuncia Adam, et al, (1992). es un tipo de distribución física orientada al producto, principalmente utilizada en sistemas de producción masivos que cumplen las mismas características; por cuanto la fabricación de grandes volúmenes del mismo producto, logra beneficiarse de este tipo de distribución, debido a su aporte en la especialización de las actividades y la eficiencia.

Cabe enunciar que la línea de ensamble esta compuesta por una serie de aspectos, entre los que se cuentan, que de acuerdo con Mejía (2005): las tareas, que consisten en un conjunto de elementos básicos e indivisibles organizados en forma lógica; las estaciones, las cuales están compuestas por uno o más operadores o máquinas donde todos realizan las mismas tareas; y el tiempo de ciclo, que es la carga de trabajo máxima que debe ser asignada a una estación de trabajo, de tal forma que la línea pueda cumplir con los requisitos de la demanda del producto. El procesamiento de todas las tareas que comprenden las diferentes operaciones en las estaciones de trabajo de la línea permite la transformación de la materia prima en el producto logrando así cubrir la demanda del mercado.

El problema de balanceo de una línea de ensamble es un clásico de la Ingeniería Industrial y se han presentado muchas exploraciones al respecto; siguiendo a Capacho et al. (2004), éste hace referencia a distribuir las tareas necesarias para ensamblar un producto a través del conjunto de estaciones que componen la línea de ensamble, existen diferentes objetivos que se pueden lograr como disminuir el número de tareas, o minimizar el tiempo de ciclo, pero todos ellos apuntan a incrementar la eficiencia de la línea; así mismo se hace necesario respetar una serie de restricciones como son las relaciones entre las tareas.

El algoritmo COMSOAL (Computer Method of Sequencing Operations for Assembly Lines) según Panneerselvam (2006) es un procedimiento que genera un gran número de soluciones factibles usando un método de simulación, pudiendo escoger la solución que mas se ajusta a las necesidades de la problemática. Siendo estas, la asignación de todos los predecesores inmediatos y que los tiempos de procesamiento sean menores o iguales al tiempo de ciclo que esta pendiente por asignar.

\section{Marco referencial teórico}

En cuanto al estado actual del conocimiento relacionado al balanceo de mallas curriculares o planes de estudio, Restrepo et al, (2006) utilizaron el algoritmo de Helgeson y Birnie, desarrollando soluciones para un plan de 8 semestres y uno de 10 semestres, con el mismo número de asignaturas; los autores destacan el buen desempeño de la herramienta, pues les permitió identificar ciertas debilidades en el problema analizado.

De igual forma, Aguilar et al, (2007) desarrollo un modelo matemático, el cual fue programado en HyperLingo, comparando el plan existente con el modelo propuesto que considera aspectos adicionales como una menor carga en el primer y en el último semestre; obteniendo un mejor desempeño por parte del modelo propuesto en cuanto a tiempos de ejecución, costos y carga balanceada.

En cuanto a la utilización del algoritmo COMSOAL en líneas de ensamble, Restrepo et al, (2008) consideraron un problema numérico simultáneamente como SALBP - 1 y SALBP - 2, precisando la facilidad de implementación de la herramienta y la posibilidad de obtener varias soluciones a diferencia de otros algoritmos como el RPW. De igual forma, Restrepo id, (2008) abordaron el problema de programación de vehículos con restricciones de capacidad (CVRP) como una combinación de SALBP - 1 aplicando el COMSOAL para la determinación del número de vehículos y la Heurística R para determinar la secuencia de entrega. 
La presente investigación, utiliza el algoritmo COMSOAL por considerar su aplicabilidad en la problemática planteada, su eficiencia en problemas de optimización y la posibilidad de obtener diversas soluciones permitiendo un análisis más profundo de la situación y aportando a la toma de decisiones.

El algoritmo COMSOAL ha sido ampliamente utilizado por diversos autores para la generación de aplicaciones, por cuanto su técnica es muy sencilla de aplicar y proporciona buenos resultados.

\section{Metodología}

En la figura 1 se presentan los pasos aplicados en el presente escrito, para generar el balanceo de la malla curricular del programa de Ingeniería Industrial, utilizando el algoritmo COMSOAL.

\section{Parámetros Requeridos}

El primer paso es consolidar toda la información de la malla curricular; donde las tareas son las asignaturas o cursos, el tiempo de procesamiento son los créditos de cada una de las asignaturas y los precedentes son los prerrequisitos establecidos para cada una de las asignaturas. También se debe establecer el tiempo de ciclo, el cual representa el número de créditos por semestre y a su vez el número de estaciones que son el total de semestres a cursar.

\section{Elaboración de la Lista $\mathbf{A}$}

Generar una lista A con todas las asignaturas no asignadas y sus respectivos prerrequisitos inmediatos no asignados

\section{Elaboración de la Lista B}

A partir la lista A debe generar la lista B, la cual contiene con las asignaturas que no tienen prerrequisitos inmediatos $y$ sus respectivos créditos. Las asignaturas consignadas en esta lista son candidatas para asignar a los semestres o estaciones.

\section{Elaboración de la Lista C}

La lista $C$ se elabora basada en la información proporcionada por la lista B, donde se incluyen las tareas con tiempo de proceso menor al tiempo disponible de la estación, es decir las asignaturas con carga de créditos menor que los créditos disponibles del semestre. Cuando el semestre no contenga créditos disponibles, se inicia un nuevo semestre. Parar si no existen asignaturas sin ser asignadas al semestre.

\section{Asignación de los cursos}

Seleccionar una asignatura de la lista $C$ de manera aleatoria y asignarla a la estación o semestre.

\section{Actualización}

Una vez asignado el curso al semestre, se debe actualizar la lista A eliminando el curso o tarea asignada y de igual forma como prerrequisito. También actualizar el número de créditos disponibles, el listado B y $C$

\section{Desarrollos creados por los autores, que surgen en la cons- trucción metodológica para la aplicación del COMSOAL al plan de estudios}

En la aplicación del algoritmo COMSOAL al caso de estudio fue necesario establecer algunas presunciones, como es el caso de las asignaturas de correquisitos, que son aquellas que deben tomarse al tiempo; es decir, en el mismo semestre, como por ejemplo los cursos teóricos y sus respectivos laboratorios que se agruparon en un solo curso. La Tabla 1 muestra el listado de asignaturas que componen el Plan de Estudios del Programa de

Tabla 1. Parámetros requeridos del COMSOAL

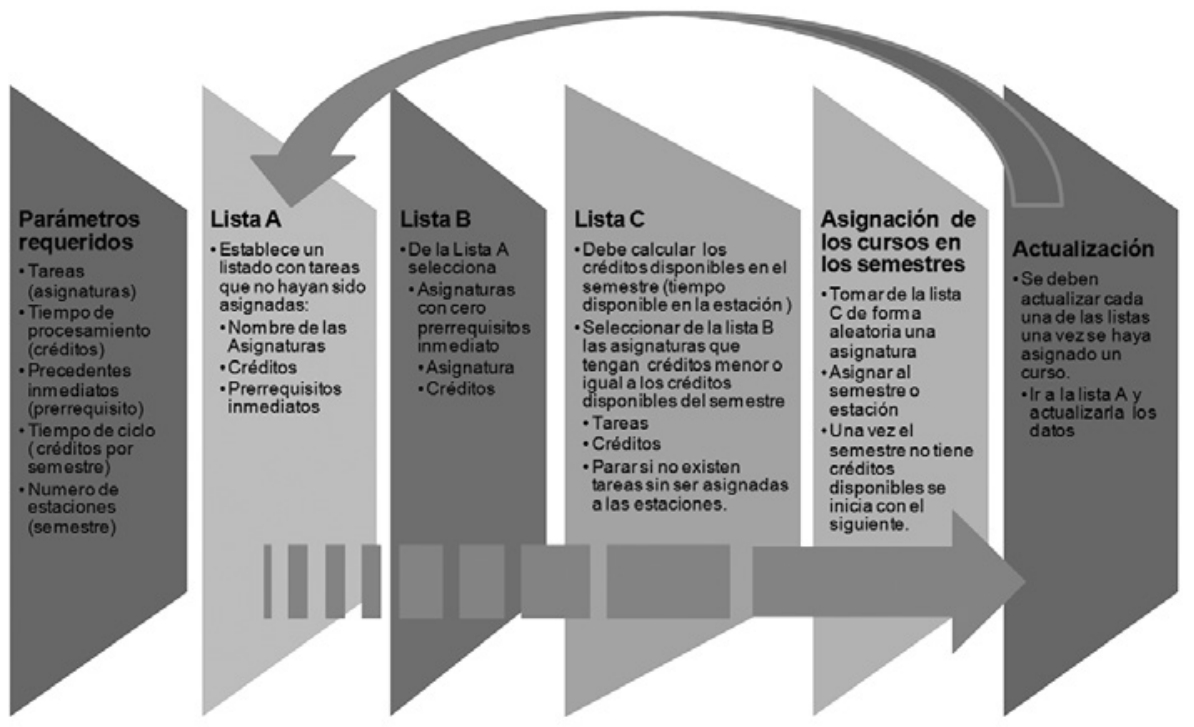

Fuente: Los autores 
Figura 1. Metodología del Balanceo de la malla Curricular utilizando COMSOAL

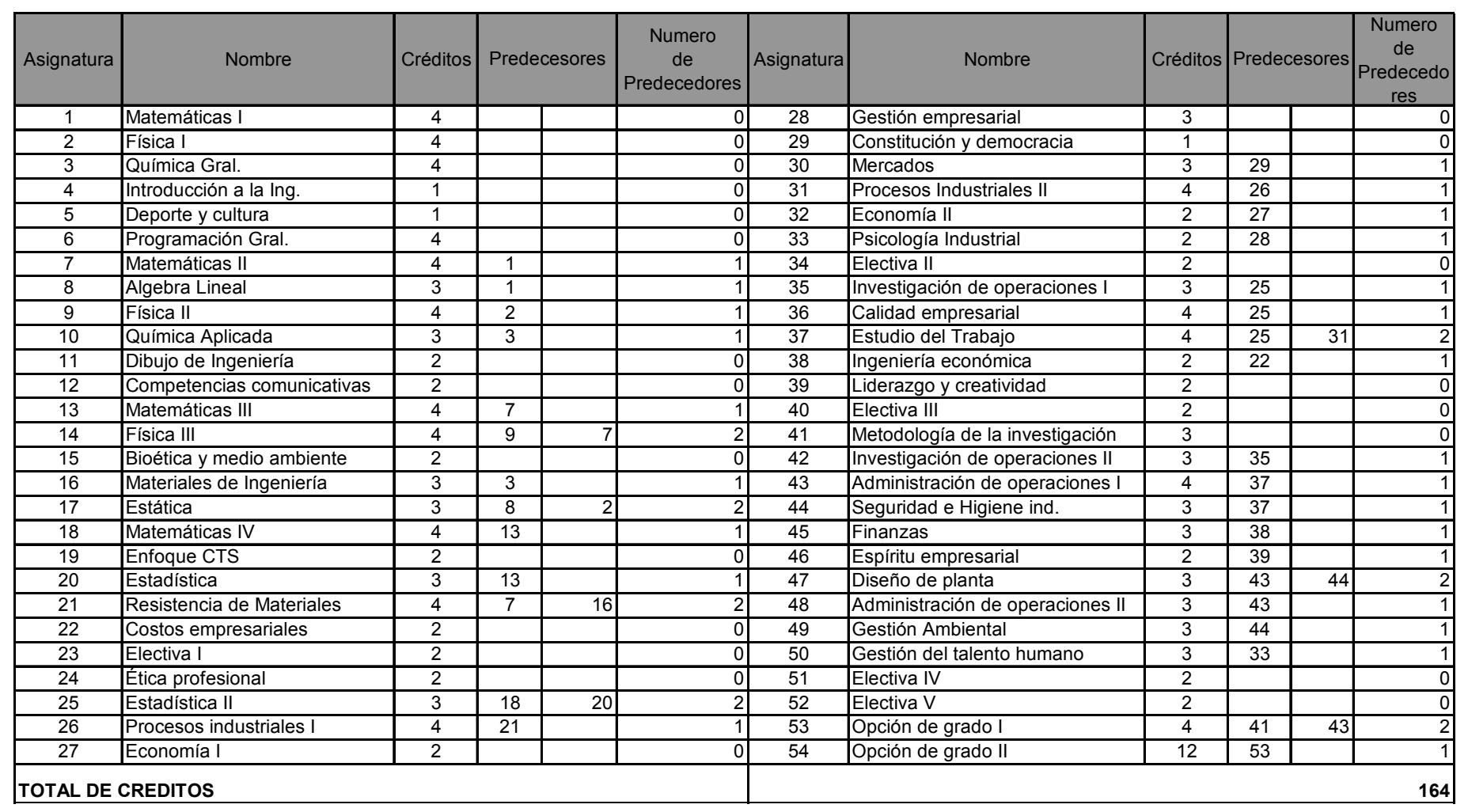

Fuente: Los autores

Ingeniería Industrial con sus concernientes créditos y la relación de prerrequisitos entre las asignaturas.

A partir de la anterior información se establecen los siguientes datos:

- Tareas o cursos a asignar son 54.

- Estaciones de trabajo o semestres son 10

- Tiempo de ciclo o créditos de cada semestre 17

A continuación se presenta la primera iteración; donde inicialmente se obtiene la primera tabla que corresponde a todas las asignaturas que no tienen prerrequisitos, de esta manera se construye la lista A, como se muestra en la tabla 2.

El siguiente paso es generar la lista B basada en la lista A, que incluye las asignaturas que no tienen precedentes. Como se observa en la tabla 3 , se cuenta con 22 asignaturas en el plan de estudios las cuales no tienen prerrequisitos y que son candidatas para ser asignadas.

Siguiendo con la metodología presentada, es necesario calcular el tiempo disponible del semestre, para este caso es igual al tiempo de ciclo que es 17 unidades. Para la generación de la lista $C$ se deben incluir las asignaturas menor o igual al tiempo disponible, para esta iteración todas las asignaturas tienen créditos menores del tiempo disponible, en la Tabla 4 se presenta el listado $C$.
Aleatoriamente de la lista C, con la ayuda de Excel, se selecciona una asignatura; en esta iteración la asignatura asignada al primer semestre fue la número 19. En la tabla 5 se presenta la primera asignatura asignada.

El siguiente paso es la actualización de las listas; cuando la asignatura 19 es asignada, se debe remitir a la lista A, eliminarla y actualizar aquellas tareas que la asignatura 19 es prerrequisito; en este caso la asignatura 19 no es prerrequisito de ningún otro curso. Cuando en la lista A no tenga ningún curso por asignar, se debe detener el algoritmo.

En la Tabla 6 se presentan los resultados del balanceo del Plan de estudios del Programa de Ingeniería Industrial, aplicando el Algoritmo COMSOAL.

\section{Análisis de resultados}

La eficiencia siguiendo a Heizer, et al., (2004) corresponde a la relación del tiempo total de las tareas y el producto del número de estaciones requeridas por el tiempo de ciclo asignado; lo que determina la cantidad de tiempo ocioso que se genera en una línea.

Como se puede observar en la tabla 6 , en los semestres $1,2,3$, 4, 6 y 10 el tiempo de ciclo de la estación o número de créditos del semestre corresponden exactamente al tiempo de ciclo esta- 
Tabla 2. Lista A del algoritmo del COMSOAL

\begin{tabular}{|c|c|c|c|c|c|c|c|c|c|}
\hline \multirow{2}{*}{$\begin{array}{c}\text { Asignatura } \\
1\end{array}$} & \multirow{2}{*}{$\begin{array}{c}\text { Créditos } \\
4\end{array}$} & \multicolumn{2}{|c|}{ Predecesores } & \multirow{2}{*}{$\begin{array}{c}\begin{array}{c}\text { Numero } \\
\text { de } \\
\text { Predecedores }\end{array} \\
0 \\
\end{array}$} & \multirow{2}{*}{$\begin{array}{c}\text { Asignatura } \\
28\end{array}$} & \multirow{2}{*}{$\begin{array}{c}\text { Créditos } \\
3\end{array}$} & \multicolumn{2}{|c|}{ Predecesores } & \multirow{2}{*}{$\begin{array}{c}\begin{array}{c}\text { Numero } \\
\text { de } \\
\text { Predecedores }\end{array} \\
0\end{array}$} \\
\hline & & & & & & & & & \\
\hline 2 & 4 & & & 0 & 29 & 1 & & & 0 \\
\hline 3 & 4 & & & 0 & 30 & 3 & 29 & & 1 \\
\hline 4 & 1 & & & 0 & 31 & 4 & 26 & & 1 \\
\hline 5 & 1 & & & 0 & 32 & 2 & 27 & & 1 \\
\hline 6 & 4 & & & 0 & 33 & 2 & 28 & & 1 \\
\hline 7 & 4 & 1 & & 1 & 34 & 2 & & & 0 \\
\hline 8 & 3 & 1 & & 1 & 35 & 3 & 25 & & 1 \\
\hline 9 & 4 & 2 & & 1 & 36 & 4 & 25 & & 1 \\
\hline 10 & 3 & 3 & & 1 & 37 & 4 & 25 & 31 & 2 \\
\hline 11 & 2 & & & 0 & 38 & 2 & 22 & & 1 \\
\hline 12 & 2 & & & 0 & 39 & 2 & & & 0 \\
\hline 13 & 4 & 7 & & 1 & 40 & 2 & & & 0 \\
\hline 14 & 4 & 9 & 7 & 2 & 41 & 3 & & & 0 \\
\hline 15 & 2 & & & 0 & 42 & 3 & 35 & & 1 \\
\hline 16 & 3 & 3 & & 1 & 43 & 4 & 37 & & 1 \\
\hline 17 & 3 & 8 & 2 & 2 & 44 & 3 & 37 & & 1 \\
\hline 18 & 4 & 13 & & 1 & 45 & 3 & 38 & & 1 \\
\hline 19 & 2 & & & 0 & 46 & 2 & 39 & & 1 \\
\hline 20 & 3 & 13 & & 1 & 47 & 3 & 43 & 44 & 2 \\
\hline 21 & 4 & 7 & 16 & 2 & 48 & 3 & 43 & & 1 \\
\hline 22 & 2 & & & 0 & 49 & 3 & 44 & & 1 \\
\hline 23 & 2 & & & 0 & 50 & 3 & 33 & & 1 \\
\hline 24 & 2 & & & 0 & 51 & 2 & & & 0 \\
\hline 25 & 3 & 18 & 20 & 2 & 52 & 2 & & & 0 \\
\hline 26 & 4 & 21 & & 1 & 53 & 4 & 41 & 43 & 2 \\
\hline 27 & 2 & & & 0 & 54 & 12 & 53 & & 1 \\
\hline Total & & & & & & & & & 164 \\
\hline
\end{tabular}

Fuente: Los autores

Tabla 3. Lista B del algoritmo del COMSOAL.

\begin{tabular}{|c|c|c|c|c|c|}
\hline Asignatura & Créditos & $\begin{array}{c}\text { Numero } \\
\text { de Predecedores }\end{array}$ & Asignatura & Créditos & $\begin{array}{c}\text { Numero } \\
\text { de Predecedores }\end{array}$ \\
\hline 1 & 4 & 0 & 23 & 2 & 0 \\
\hline 2 & 4 & 0 & 24 & 2 & 0 \\
\hline 3 & 4 & 0 & 27 & 2 & 0 \\
\hline 4 & 1 & 0 & 28 & 3 & 0 \\
\hline 5 & 1 & 0 & 29 & 1 & 0 \\
\hline 6 & 4 & 0 & 34 & 2 & 0 \\
\hline 11 & 2 & 0 & 39 & 2 & 0 \\
\hline 12 & 2 & 0 & 40 & 2 & 0 \\
\hline 15 & 2 & 0 & 41 & 3 & 0 \\
\hline 19 & 2 & 0 & 51 & 2 & 0 \\
\hline 22 & 2 & 0 & 52 & 2 & 0 \\
\hline
\end{tabular}

Fuente:Los autores.

Tabla 4. Lista $C$ del algoritmo del COMSOAL

\begin{tabular}{|c|c|c|c|c|c|}
\hline Asignatura & Créditos & $\begin{array}{c}\text { Numero } \\
\text { de Predecedores }\end{array}$ & Asignatura & Créditos & $\begin{array}{c}\text { Numero } \\
\text { de Predecedores }\end{array}$ \\
\hline 1 & 4 & 0 & 23 & 2 & 0 \\
\hline 2 & 4 & 0 & 24 & 2 & 0 \\
\hline 3 & 4 & 0 & 27 & 2 & 0 \\
\hline 4 & 1 & 0 & 28 & 3 & 0 \\
\hline 5 & 1 & 0 & 29 & 1 & 0 \\
\hline 6 & 4 & 0 & 34 & 2 & 0 \\
\hline 11 & 2 & 0 & 39 & 2 & 0 \\
\hline 12 & 2 & 0 & 40 & 2 & 0 \\
\hline 15 & 2 & 0 & 41 & 3 & 0 \\
\hline 19 & 2 & 0 & 51 & 2 & 0 \\
\hline 22 & 2 & 0 & 52 & 2 & 0 \\
\hline
\end{tabular}

Fuente: Los autores 
Tabla 5. Asignación de Cursos.

\begin{tabular}{|c|c|}
\hline \multicolumn{2}{|c|}{ ESTACION 1 } \\
\hline \multicolumn{2}{|c|}{ SEMESTRE 1 } \\
\hline Asignaturas & Creditos asignados \\
\hline Creditos Totales 17 & \\
\hline & Creditos disponible 13 \\
\hline
\end{tabular}

Fuente: Los autores

Tabla 6. Balanceo del Plan de estudios propuesto con el algoritmo COMSOAL.

\begin{tabular}{|c|c|c|c|}
\hline \multicolumn{2}{|c|}{ ESTACION 1 } & \multicolumn{2}{c|}{ ESTACION 2 } \\
\hline \multicolumn{2}{|c|}{ SEMESTRE 1 } & \multicolumn{2}{c|}{ SEMESTRE 2 } \\
\hline Asignaturas & Creditos asignados & Asignaturas & Creditos asignados \\
\hline $19,4,12,15,6,3,34$ & $2+1+2+2+4+4+2$ & $39,10,46,27,29,23,1,5$ & $2+3+2+2+1+2+4+1$ \\
\hline Creditos Totales 17 & Creditos disponible 0 & Creditos Totales 17 & Creditos disponible 0 \\
\hline
\end{tabular}

\begin{tabular}{|c|l|l|l|}
\hline \multicolumn{2}{|c|}{ ESTACION 3 } & \multicolumn{2}{c|}{ ESTACION 4 } \\
\hline \multicolumn{2}{|c|}{ SEMESTRE 3 } & \multicolumn{2}{c|}{ SEMESTRE 4 } \\
\hline \multicolumn{1}{|c|}{ Asignaturas } & Creditos asignados & Asignaturas & Creditos asignados \\
\hline $2,11,18,28,32,40$ & $4+2+4+3+2+2$ & $41,51,33,7,9,22$ & $3+2+2+4+4+2$ \\
\hline Creditos Totales 17 & Creditos disponible 0 & Creditos Totales 17 & Creditos disponible 0 \\
\hline
\end{tabular}

\begin{tabular}{|l|l|l|l|}
\hline \multicolumn{2}{|c|}{ ESTACION 5 } & \multicolumn{2}{c|}{ ESTACION 6} \\
\hline \multicolumn{2}{|c|}{ SEMESTRE 5 } & \multicolumn{2}{c|}{ SEMESTRE 6} \\
\hline Asignaturas & \multicolumn{1}{|c|}{ Creditos asignados } & \multicolumn{1}{c|}{ Asignaturas } & \multicolumn{1}{c|}{ Creditos asignados } \\
\hline $8,16,52,30,13,45$ & $3+3+3+4+3$ & $14,21,20,17,25$ & $4+4+3+3+3$ \\
\hline Creditos Totales 17 & Creditos disponible 1 & Creditos Totales 17 & Creditos disponible 0 \\
\hline
\end{tabular}

\begin{tabular}{|c|c|c|c|}
\hline \multicolumn{2}{|c|}{ ESTACION 7 } & \multicolumn{2}{c|}{ ESTACION 8 } \\
\hline \multicolumn{2}{|c|}{ SEMESTRE 7 } & \multicolumn{2}{c|}{ SEMESTRE 8 } \\
\hline \multicolumn{1}{|c|}{ Asignaturas } & Creditos asignados & Asignaturas & Creditos asignados \\
\hline $38,24,26,35,37$ & $2+2+4+3+4$ & $31,36,43,44$ & $4+4+4+3$ \\
\hline Creditos Totales 17 & Creditos disponible 2 & Creditos Totales 17 & Creditos disponible 2 \\
\hline
\end{tabular}

\begin{tabular}{|c|c|c|c|}
\hline \multicolumn{2}{|c|}{ ESTACION 9} & \multicolumn{2}{|c|}{ ESTACION 10} \\
\hline \multicolumn{2}{|c|}{ SEMESTRE 9} & \multicolumn{2}{|c|}{ SEMESTRE 10} \\
\hline Asignaturas & Creditos asignados & Asignaturas & Creditos asignados \\
\hline $42,48,47,49,53$ & $3+3+3+3+4$ & $54,52,50$ & $12+2+3$ \\
\hline Creditos Totales 17 & Creditos disponible 1 & Creditos Totales 17 & Creditos disponible 0 \\
\hline
\end{tabular}

Fuente:Los autores.

Tabla 7. Eficiencia del Plan de estudios propuesto con el algoritmo COMSOAL.

\begin{tabular}{|l|r|r|}
\cline { 2 - 3 } \multicolumn{1}{c|}{} & Eficiencia & Créditos Ociosos \\
\hline Semestre 1 & $100 \%$ & 0 \\
\hline Semestre 2 & $100 \%$ & 0 \\
\hline Semestre 3 & $100 \%$ & 0 \\
\hline Semestre 4 & $100 \%$ & 0 \\
\hline Semestre 5 & $94 \%$ & 1 \\
\hline Semestre 6 & $100 \%$ & 0 \\
\hline Semestre 7 & $88 \%$ & 2 \\
\hline Semestre 8 & $88 \%$ & 2 \\
\hline Semestre 9 & $94 \%$ & 1 \\
\hline Semestre 10 & $100 \%$ & 0 \\
\hline
\end{tabular}

Fuente:Los autores. 
blecido, es decir que el total de créditos correspondientes a las asignaturas de dichos semestres suman 17 créditos, lo que hace que la eficiencia de estos sea del 100\%. Para el Plan de estudio actual, con un número de créditos total de 164 y el tiempo total de las tareas de 20 créditos en los 10 periodos académicos, resulta en una eficiencia del $82 \%$. De igual forma, el Plan de estudio generado por el Algoritmo COMSOAL, con un tiempo de ciclo de 164 créditos y el tiempo total de las tareas de 17 créditos en los 10 periodos académicos, resulta en una eficiencia del 96,47\%. En la tabla 7 se muestra la eficiencia de semestre a semestre del Plan de estudios propuesto generado por el Algoritmo COMSOAL.

\section{Conclusiones y recomendaciones}

Las herramientas de optimización se convierten en un apoyo para problemas reales, más específicamente para el análisis de los planes de estudio y rediseños curriculares. La utilización de este tipo de instrumentos propios de la Ingeniería Industrial, deja al descubierto posibles debilidades o falencias en la determinación de relaciones entre asignaturas; es el caso de Matemáticas 1, la cual fue asignada por el Algoritmo en el segundo semestre, puesto que no presenta ningún prerrequisito y necesita ser cursada por los estudiantes al inicio de su carrera profesional debido a las competencias que esta proporciona. De igual forma en el caso de la asignatura Electiva 1 que pertenece al área profesional, el algoritmo la asignó en el segundo semestre la cual es una asignatura de alta complejidad y que los estudiantes no poseen las competencias para cursarla.

Los resultados arrojados por el Algoritmo COMSOAL sugieren el establecimiento de restricciones en las relaciones entre las asignaturas, forzando que estas sean cursadas teniendo competencias necesarias.

Dado que la tendencia es hacia la reducción de los semestres académicos, se recomienda aplicar este modelo para ocho semestres y comparar los resultados arrojados por el COMSOAL con otros algoritmos de balanceo de líneas.

\section{Referencias}

Adam, E y Evert, R. (1992). Administración de La producción y las operaciones: conceptos, modelos y funcionamiento. México: Pearson Educación, pp. 739.

Aguilar J., Martínez J., Cabrera M. y Nuño J. (2007). El problema de balancear un plan de estudios: un modelo matemático. $2^{\circ}$ Taller Latino Iberoamericano de Investigación de Operaciones, Acapulco, México: Guerrero.

Arcus, A. (1966). COMSOAL: A computer method of sequencing operations for assembly lines: International Journal of Production Research, pp. 259-277.

Capacho L., Pastor R. (2004). Generación de secuencias de montaje y equilibrado de líneas, Universidad Politécnica de Catalunya pp. 12.

Heizer J. y Reinder B. (2004). Principios de Administración de operaciones. México: Pearson, Educación, 5ta ed., pp. 351.

Mejía, H. (2005). Minimización de los costos totales en el problema de balanceo de línea con ciclo variable y estaciones en paralelo, pp. 2, 3,14.

Panneerselvam R. (2006). Production and operations management India: Prentice Hill, 2nd ed., pp. 188.

Restrepo, J. y Medina, P. (2008). Un problema logístico de programación de vehículos con capacidad finita. Scientia et Technica Año XIV, 38 (1), pp. 253-258.

Restrepo, J., Cruz, E. y Saracho C. (2006). Una heurística de balanceo de línea de producción aplicada a una Malla curricular. Scientia et Technica Año XII, 30, (1) pp. 267-272.

Restrepo, J., Medina, P., y Cruz, E. (2008). Problemas de balanceo de línea salbp-1 y salbp-2: un caso de estudio. Scientia et Technica Año XIV, 40, (1) pp. 105-110. 